\title{
HMD Best Paper Award - Verleihung 2018
}

\author{
Matthias Knoll • Sybille Thelen
}

Online publiziert: 22. März 2018

(C) Springer Fachmedien Wiesbaden GmbH, ein Teil von Springer Nature 2018

Der 7. HMD Best Paper Award wurde dieses Jahr im Rahmen der Multikonferenz Wirtschaftsinformatik 2018 im neuen Zentralgebäude der Leuphana Universität Lüneburg verliehen, dem spektakulären Libeskind-Bau an der Universitätsallee. Das vor fast genau einem Jahr feierlich eröffnete Gebäude bot mit seiner herausragenden Architektur einen glanzvollen Rahmen für die Verleihung, die wie stets am Springer-Buchstand stattfand. Es hat uns sehr gefreut, dass sich auch in diesem Jahr die Preisträger aller drei Beiträge die Zeit genommen haben unserer Einladung nach Lüneburg zu folgen und dass zahlreiche interessierte Zuhörer die Preisverleihung verfolgten.

Für das Jahr 2017 wurden die folgenden Beiträge prämiert:

- Adelmeyer, M./Petrick, C./Teuteberg, F.: IT-Risikomanagement von Cloud-Dienstleistungen im Kontext des IT-Sicherheitsgesetzes, HMD - Praxis der Wirtschaftsinformatik 54 (2017), 313, S. 111-123.

$\mathrm{Zu}$ einer der sicherlich sehr kontrovers diskutierten Fragen im Rahmen der Ausrichtung des IT-Betriebs auf moderne Konzepte gehört die Frage: „Cloud Ja oder Nein?“. Dies gilt in besonderem Maße für Betreiber kritischer Infrastrukturen. Der Beitrag greift die Problemstellung vor dem Hintergrund des IT-Sicherheitsgesetzes und weiterer rechtlicher Vorgaben auf. Die komplexe Theorie wird strukturiert und verständlich dargestellt. Den Kern dieses Beitrags bilden zum einen die für die Praxis hilfreichen Tabellen mit Anforderungen an Betreiber sowie eine Syste-

\footnotetext{
M. Knoll ( $\bowtie)$

Hochschule Darmstadt, Darmstadt, Deutschland

E-Mail: matthias.knoll@h-da.de

S. Thelen

Springer Vieweg, Wiesbaden, Deutschland

E-Mail: sybille.thelen@springer.com
} 
matisierung von Szenarien, in denen die Rollen der Beteiligten definiert sind, zum anderen die Handlungsempfehlungen für Betreiber von kritischen Infrastrukturen und Cloud-Dienstleister.

- Rohmann, S./Schumann, M.: Best Practices für die Mitarbeiter-Partizipation in der Produktentwicklung, HMD - Praxis der Wirtschaftsinformatik 54 (2017), 316, S. 575-590.

Fragen der Partizipation, insbesondere unter Einbeziehung von Social Media und anderen aktuellen IT-Lösungen, werden häufig vorschnell als „Spielerei“ verurteilt, Potentiale damit verschenkt. Der Beitrag thematisiert auch diesen Aspekt im Rahmen einer kritischen Auseinandersetzung mit den erkannten Problemstellungen. Eine differenzierte und aufschlussreiche Beschreibung von Partizipationsoptionen bildet den theoretischen Kern des Beitrags. Aus Sicht des Praxiseinsatzes besonders interessant sind Zitate zu Best Practices und konkreten Handlungsempfehlungen. Zudem wird ein ,,altes“ Problem, insbesondere in Europa kritisch reflektiert: die Frage der Geheimhaltung versus dem Konzept der Open Innovation.

- Spottke, B.: Was Unternehmen von der Videospieleindustrie für die Gestaltung der Digital Customer Experience lernen können. Eine Analyse der Plattform „Steam“, HMD - Praxis der Wirtschaftsinformatik 54 (2017), 317, S. 727-740.

Beispiele für misslungene Digital Customer Experience gibt es viele. Umso naheliegender, verdienstvoller und interessanter ist es, vielversprechende und bewährte Ansätze interdisziplinär auf andere Branchen zu übertragen. Der Beitrag greift diesen Gedanken auf und findet in der Videospiele-Plattform „Steam“ interessante, gut übertragbare Ansätze, die zunächst in Form von Gestaltungsebenen für Services und das Serviceportfolio, die Technologie und - besonders wichtig die sozialen Strukturen beschrieben werden. Den Kern des Beitrags bilden darauf aufbauend konkrete Handlungsempfehlungen und Illustrationen dieser Empfehlungen am Beispiel sehr unterschiedlicher Branchen (Automobil, Streaming und Versicherungen).

Abb. 1 zeigt die angereisten Autoren und (Gast-)Herausgeber sowie Mitarbeiter des Springer-Verlags.

Es werden nun also bereits im siebten Jahr alljährlich die drei besten Beiträge eines Jahrgangs der Zeitschrift „HMD - Praxis der Wirtschaftsinformatik“ mit dem „HMD Best Paper Award“ ausgezeichnet. Das HMD-Herausgebergremium wählt die prämierten Beiträge aus und orientiert sich dabei an folgenden bewährten Kriterien:

- Zielgruppenadressierung

- Handlungsorientierung und Nachhaltigkeit

- Originalität und Neuigkeitsgehalt

- Erkennbarer Beitrag zum Erkenntnisfortschritt

- Nachvollziehbarkeit und Überzeugungskraft

- Sprachliche Lesbarkeit und Lebendigkeit

Mit dem „HMD Best Paper Award“ möchten wir allen Leserinnen und Lesern eine Sammlung herausragender Beiträge präsentieren und den Autorinnen und Autoren, denen wir diese Beiträge zu verdanken haben, zugleich unsere besondere Anerkennung zeigen. Im Rahmen der Preisverleihung erhält jeder Preisträger eine 


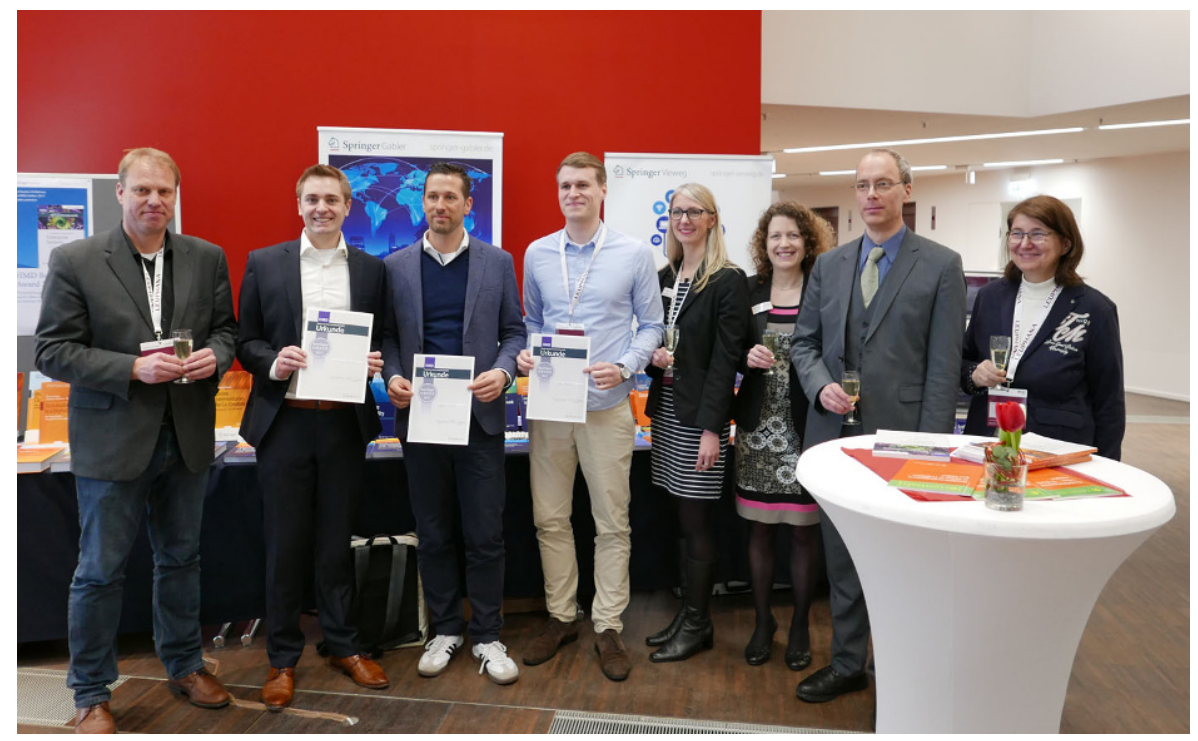

Abb. 1 Verleihung HMD Best Paper Award: (v.1.n.r) C. Lattemann, S. Rohmann, B. Spottke, M. Adelmeyer, S. Kathke, S. Thelen, M. Knoll, S. Strahringer

individuelle Urkunde und seit 2013 die Möglichkeit, seinen Beitrag in der „Essentials“-Reihe von Springer in überarbeiteter, meist ergänzter oder originaler Fassung $\mathrm{zu}$ veröffentlichen.

Die HMD, die seit 2014 bei Springer Vieweg erscheint, blickt auf eine sehr lange Geschichte und Tradition zurück. Unter dem Namen „Handbuch der maschinellen Datenverarbeitung “wurde sie im Oktober 1964 als Loseblattsammlung erstmals ausgeliefert. Seit 1998 erscheint sie unter dem Titel „HMD - Praxis der Wirtschaftsinformatik“. In jährlich sechs Schwerpunktheften tragen sowohl Wissenschaftler als auch Praktiker durch ihre unterschiedlichen Blickwinkel zu einer differenzierten Betrachtung der jeweiligen Schwerpunktthemen bei.

So wie wir die Tradition der HMD - Praxis der Wirtschaftsinformatik fortsetzen, ist mittlerweile auch der HMD Best Paper Award zu einer Tradition geworden. Wir möchten Sie einladen, Beiträge einzureichen und sind uns sicher, dass uns die exzellenten Beiträge dann auch künftig nicht ausgehen werden. Es bleibt eine nicht ganz einfache, aber dafür sehr schöne Aufgabe, die besten drei Beiträge eines Jahrgangs zu finden und auszuzeichnen.

In diesem Sinne freuen wir uns bereits jetzt auf Ihre Beiträge in den Heften des aktuellen Jahres und die Preisverleihung 2019 in Siegen.

Die Herausgeberinnen und Herausgeber der HMD und der Verlag 